\title{
Drug Accountability Treatment Dispensation Date
}

National Cancer Institute

\section{Source}

National Cancer Institute. Drug Accountability Treatment Dispensation Date. NCI

Thesaurus. Code C83166.

The drug accountability information that indicates the date that the study treatment was dispensed. 